\title{
Introduction to Electrochemical Impedance Spectroscopy as a Measurement Method for the Wetting Degree of Lithium-Ion Cells
}

\author{
Florian J. Günter, (1), Jan Bernd Habedank, ${ }^{1}{ }^{1}$ David Schreiner, ${ }^{1}$ Tobias Neuwirth, ${ }^{2}{ }^{2}$ \\ Ralph Gilles, ${ }^{2}$ and Gunther Reinhart ${ }^{1}$
}

${ }^{I}$ Technical University of Munich, Institute for Machine Tools and Industrial Management, 85748 Garching, Germany

${ }^{2}$ Technical University of Munich, Heinz, Maier-Leibnitz Zentrum, 85748 Garching, Germany

\begin{abstract}
In the production process chain of lithium-ion battery cells, the filling, consisting of dosing and wetting steps, of the cell and its components with electrolyte liquid is eminent for the final product quality and costs. To reduce the unnecessary wetting duration between filling and formation, and thereby the production costs, a measurement method for the wetting progress is necessary. In this paper, electrochemical impedance spectroscopy (EIS) as a well-established technique is used for the first time to quantify the wetting degree of batteries during cell production. The experimental data of the EIS acquired during the dosing and subsequent wetting process is correlated to images recorded by in situ neutron radiography. Results show that the impedance of the battery cells strongly depends on the wetting degree of the cell assembly and can thus be used to determine the fully wetted state enabling faster processing.

(C) The Author(s) 2018. Published by ECS. This is an open access article distributed under the terms of the Creative Commons Attribution 4.0 License (CC BY, http://creativecommons.org/licenses/by/4.0/), which permits unrestricted reuse of the work in any medium, provided the original work is properly cited. [DOI: 10.1149/2.0081814jes]

(cc) BY
\end{abstract}

Manuscript submitted July 3, 2018; revised manuscript received September 17, 2018. Published October 17, 2018.

Lithium-ion batteries (LIB) as electrochemical energy storage systems are a key-technology to substitute fossil fuels and use renewable resources due to its low weight, high energy densitiy and long service life. These batteries have established a dominant role in consumer electronic markets over the last 15 years and triggered the success of mobile devices like cell phones and portable computers. Hence, the electric automotive transportation and renewable energy storage markets are expected to be strongly driven forward by LIB.

The goal of higher energy density in automotive applications can be achieved by reducing the weight of inactive materials, like current collector foils, casing components or separators per cell. This promotes the trend toward a larger cell size and format ${ }^{2}$ as well as thicker electrodes. However, large-format cells present challenges to the production processes like the filling with liquid electrolyte. To ensure reliable operation and high capacity, all cavities and pores of the electrodes and separators have to be wetted before starting the formation cycling. Otherwise, the risk of an inhomogeneously built solid electrolyte interphase (SEI) in these first charge and discharge cycles exists. As a result, strong local layer thickness fluctuations can induce partial layer detachments and electrical resistance extremes that have a negative effect on the capacity and service life of the cell. The increase in the number of layers, electrode thickness and surface area per cell decelerates the time-intensive wetting of the cell components with electrolyte even further. ${ }^{3}$ The quality limiting production steps filling, consisting of dosing and wetting steps, and formation cycling present a bottleneck in the process chain and therefore influence the production costs of a cell. In industrial cell production, multiple periodic wetting and formation cycles are performed resulting in an overall duration of 1.5 to 3 weeks. This implies a significant expense as tens of thousands of cycles as well as storage rooms are needed to manage required throughput rates. ${ }^{4}$

One way to decrease the costs of LIB is to accelerate the wetting process. Weydanz et al. ${ }^{5}$ showed that filling under vacuum down to 100 mbar accelarates the production step significantly. The wetting state is defined as surfaces and cavities of electrodes an separator wetted and saturated with electrolyte compared to the total of surfaces and cavities of the cell assembly. The filling of cavities, as inner surfaces of the media, can be described as microscopic wetting, whereas the wetting of (macroscopic) surfaces can be regarded as superficial wetting. The latter is shown schematically in Figure 1 depending on the rise of the electrolyte liquid height $h_{e l}$ during the filling process. However, to date, there are no industrially established measuring methods to determine the wetting state of the cell. As a result, the required duration

${ }^{\text {z} E-m a i l: ~ f l o r i a n . g u e n t e r @ i w b . m w . t u m . d e ~}$ for the wetting progress is merely estimated based on experience and validated with cell tests.

Knoche et al. ${ }^{6}$ were able to visualize the filling process in operando for the first time by neutron radiography (NR). NR is an alternative transmission method to X-ray imaging using neutron radiation for the image acquisition by transmitting through an object to visualize its internal structure. ${ }^{7}$ While X-rays mainly interact with the electron shell of atoms, neutrons interact with the nuclei. As a result of this physical principle of interaction, neutrons are strongly attenuated by a few light elements like hydrogen, lithium or boron whereas a few metallic elements like aluminum hardly alter the beam intensity. Therefore the electrolyte liquid by means of its hydrogen groups can be detected. ${ }^{8}$ In the NR image, the electrolyte liquid appears darker than other components and the not wetted parts of the cell appear brighter. Thus, the electrolyte distribution in the cross-sectional area of the cell can be visualized over time. NR can also be used to detect gas entrapments between the layers and to give insight in process phenomena of filling. ${ }^{6}$

Despite those achievements the challenge of determining the wetted state of LIB remains prominent for the production industry. Limited access to NR as well as the multitude of available cells on the market and their components complicate the detailed prediction of the wetting degree and thereby the optimal formation start time. Cellspecific detection would be ideal to initiate the first charging cycle of the cell at the right time.

The wetting influences the impedance $Z$ of the cell. ${ }^{9}$ The impedance is the ratio between complex voltage $u$ and complex current $i$

$$
Z=\frac{u(t)}{i(t)}=\frac{u_{0} \cdot \sin \left(\omega t+\phi_{1}\right)}{i_{0} \cdot \sin \left(\omega t+\phi_{2}\right)}=Z_{0} \cdot \frac{\sin \left(\omega t+\phi_{1}\right)}{\sin \left(\omega t+\phi_{2}\right)},
$$

with $\omega$ as radial frequency and phase angle $\phi_{i}$. It demonstrates the ability of a circuit to resist the flow of electrical current, which is represented by the real part. Additionally it reflects the ability of a circuit to store electrical energy, which is represented in the imaginary term. Thus, impedance can be defined as a complex resistance

$$
Z=Z_{0}(\cos (\Delta \phi)+j \sin (\Delta \phi))
$$

encountered when current flows through a circuit composed of various resistors, capacitors and inductors. ${ }^{10}$ Electrochemical Impedance Spectroscopy (EIS) is a powerful non-destructive technique to determine the impedance of a LIB by applying a current (voltage) signal and measuring the voltage (current) response. The resulting response will have the same frequency, but a different phase angle and amplitude. If the excitation signal is voltage (current), the term potentiostatic (galvanostatic) is used, whereby the potential of the open circuit voltage is 


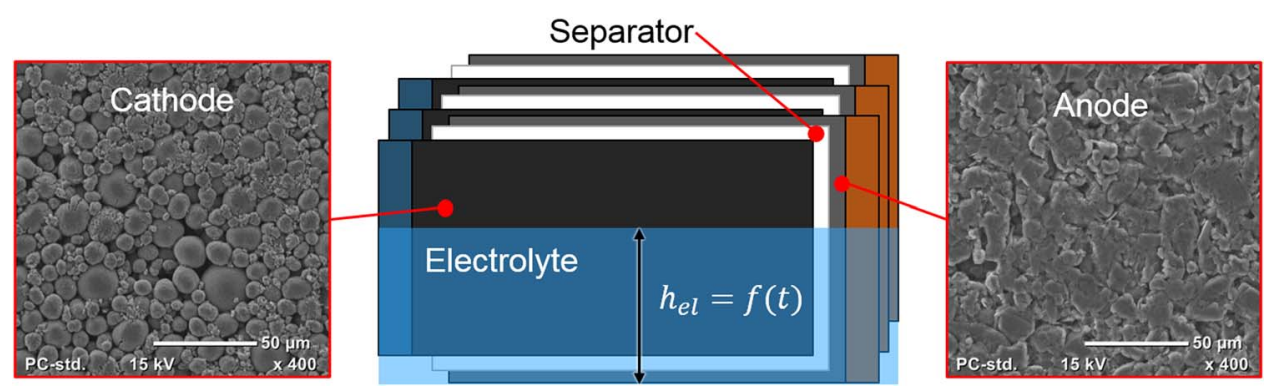

Figure 1. Schematic illustration of the rising electrolyte of a multilayer cell assembly during the filling process with scanning electron microscope (SEM) images of the unstructured NCM111 Cathode (left) and the unstructured Graphite-PVDF(95:5) Anode (right) taken at the Chair of Technical Electrochemistry (TUM).

optimal to minimize DC currents. ${ }^{11}$ The polarization applied has to be low enough so that linear conditions are maintained and the polarization increases linearly with the input signal, which can either be current (galvanostatic) or voltage (potentiostatic). ${ }^{12}$ From the measured complex cell impedance, it is possible to examine and qualitatively determine several processes of an electrochemical system. ${ }^{13,14}$ The time constants for these processes differ, so that their features show up at different frequencies in the EIS spectra. ${ }^{11}$ From high (approx. $>10^{5} \mathrm{~Hz}$ ) to low frequencies (approx. $<10^{0} \mathrm{~Hz}$ ) the transfer processes can be interpreted as followed:

I. The fastest mass transfer process and therefore visible at high frequencies in the Nyquist plot is $\mathrm{Li}^{+}$-diffusion within the electrolyte liquid through the pores of electrodes and separator resulting in the electrolyte resistance. Inductive reactances $L$ through electronic conduction in metallic elements in cell and wires shift the impedance spectrum along the imaginary axis as shown in Figure 2. The electrical resistances of the cell components together with the conductivity of the electrolyte result in the ohmic resistance $R_{H F R}$ of the cell at the zero-crossing of the real axis in the Nyquist Plot. ${ }^{15}$

II. This intersection is followed by two semi-circles. They represent ion migration through a surface layer covering the active material particles of the electodes (the SEI) ${ }^{16}$ as well as the interfacial charge transfer between electrolyte and electrodes ${ }^{17}$

$$
\mathrm{Li}^{+}+\mathrm{e}^{-} \rightleftarrows \mathrm{Li}
$$

and thus, ion transition of the double layer capacity (solid solution interphase). They are strongly dependant on temperature ${ }^{12}$ and state of charge (SOC) of the cell. With increasing SOC it gets harder to separate the semi-circles from another and the impedance of the cell decreases. ${ }^{18}$ The second (mid to low frequency) semi-circle depends additionally on the state of health (SOH) of the cell and continuously increases during cycling. ${ }^{19}$ This is attributed to structural defects in the electrode materials and the increase in the SEI layer thickness during cycling since its formation with the first charging of the cell.

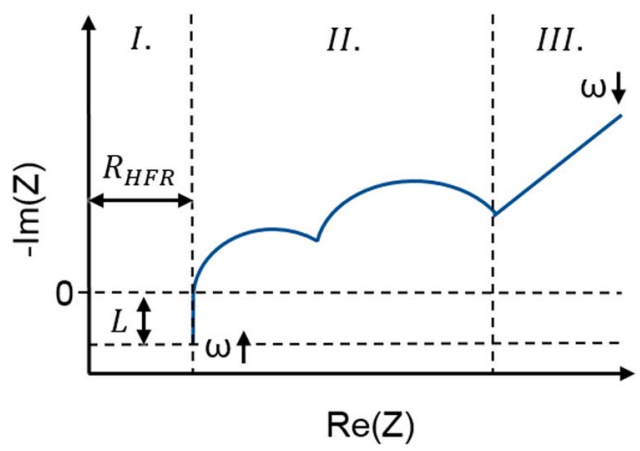

Figure 2. Schematic Nyquist plot of the EIS of a LIB.
III. The slowest mass transfer process is the solid-state $L i$-diffusion in the active material of the electrodes at very low frequencies. ${ }^{20}$ When the concentration gradient vanishes in the solid material, this section transforms into a capacitive behavior. ${ }^{21}$ But for LIBresearch, it is often not observed due to the very low frequencies at which the effect occurs. ${ }^{22}$

So far EIS has been used to measure various characteristics of LIB and its cell components. In ${ }^{19}$ the wettability of porous electrodes for LIB was examined and it was observed for the first time that the impedance varies over time after dosing electrolyte. Therefore, EIS as a measurement method for the wetting state based on first experiments is presented. The electrochemical measurements are compared with the visual data obtained by NR to verify the plausibility of the results. The following assumptions can be made for EIS used during the filling process of LIB:

1. Without the first charging cycles, no SEI has formed, which is equivalent to a layer thickness close to zero.

2. The cell is supposed to have the SOC of $0 \%$, since it has not yet been electrically charged.

3. The high-frequency resistance (HFR) of the cell $R_{H F R}$ is assumed to decrease with increasing wetting state as the electrolyte fluid allows ion exchange of the cell components.

\section{Experimental}

Sample preparation.-The pouch bag cells, consisting of 5 anodes (graphite-PVDF) and 4 cathodes (NCM111) were produced at the Technical University of Munich on semi-automated machines. Electrode production was performed in a cleanroom (ISO 6) and cell assembly in a dry room with a dew point less than $-55^{\circ} \mathrm{C}$. The anode ink was prepared by mixing 95 wt $\%$ graphite (SGL) and 5 $\mathrm{wt} \%$ polyvinylidene difluoride (PVDF) with 1-methyl-2-pyrrolidone (NMP, Sigma Aldrich) in a multi-step mixing process. ${ }^{23}$ The cathode ink contained $96 \mathrm{wt} \% \mathrm{LiNi}_{1 / 3} \mathrm{Co}_{1 / 3} \mathrm{Mn}_{1 / 3} \mathrm{O}_{2}$ (NCM111, BASF), $2 \mathrm{wt} \%$ PVDF and $2 \mathrm{wt} \%$ carbon (C65, Timical). Powder and solvent were mixed in a planetary centrifugal mixer. The resulting inks were both coated onto metallic current collectors in a roll to roll doctor blade process and dried in an infrared drying line. The coating was then repeated for the other side of the foil. For balancing the area, the specific capacity of the anode $\left(7.3 \mathrm{mg} \mathrm{cm}^{-2}\right.$ graphite per side) was matched to around $120 \%$ of the cathode with $14.4 \mathrm{mg} \mathrm{cm}^{-2} \mathrm{NCM} 111$ per side. To reduce the porosity from $50 \%$ to $30 \%$, the electrode coils were calendered at $120^{\circ} \mathrm{C}$ roller temperature. The resulting structure and the pores of anode and cathode, which influence the wettability of the components and thereby affect the filling process, ${ }^{24}$ are shown in Figure 1. To achieve different wetting behavior of the cells, the electrodes for cell B were structured with a laser beam method, which led to different mass and surface of the active material. Further details on this process as well as its the influence on the wetting behavior can be found in Ref. 25. Subsequently, electrode sheets were separated in a remote laser cutting process to a format of $101 \mathrm{~mm} \times 73 \mathrm{~mm}$ (cathode) and $104 \mathrm{~mm} \times 76 \mathrm{~mm}$ (anode). The commercial separator 
a)
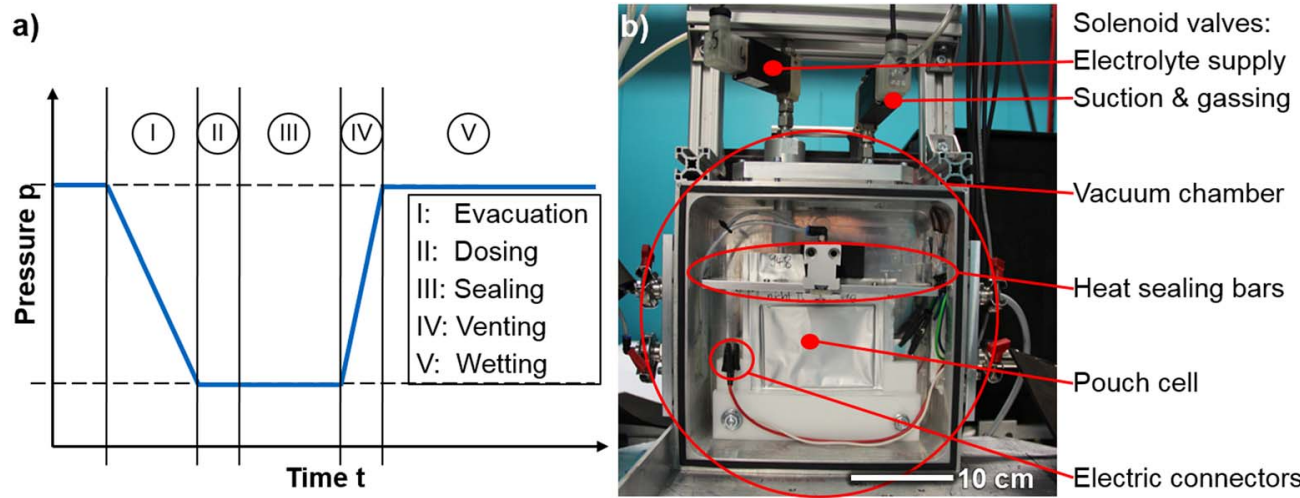

Figure 3. a) Filling process: course of pressure, b) Section of the filling station at the neutron radiography instrument ANTARES of MLZ.

(Celgard 2325) was z-folded between the electrodes to ensure electrical insulation and wrapped around the cell stack for mechanical stability. The tabs and current collector foils were joined using ultrasonic welding and the finished cell stack was packaged into the flexible pouch with a deep-drawn pocket, leaving one side open for the electrolyte filling. The details of the filling procedure will be explained in Filling process section.

Experimental setup.-The experiments were performed at the ANTARES instrument operated at the Heinz Maier-Leibnitz Zentrum (MLZ) in Garching, Germany. Further details on this instrument can be found in Ref. 26. An automated filling station, described in the following, was placed in the neutron beam close to the detector. The ambient air as well as the electrolyte temperature was kept constant at $25^{\circ} \mathrm{C}$ and the ambient pressure was approx. 950 mbar.

Filling station.-The automatic mobile filling station shown in Figure 3 (right) consists of a self-made aluminum vacuum chamber, an MD 4 NT VARIO vacuum pump from Vacuubrand, a sealing system, Bürkert solenoid valves and a dosing device. The chamber was equipped with a workpiece carrier to fix the sample in a vertical position in which the cell components are perpendicular to the neutron beam. The sealing system had two pneumatic heat sealing bars and a gate to an impulse transmitter. The dosing device was composed of an interface and the Dosino 800 from Metrohm. The electrolyte was introduced into the chamber via a vertical filling nozzle, which was integrated in the chamber and connected by a valve to the dosing device. Two tubes for opening and closing the bars by compressed air and six electric connector cables (two for the heat sealing system and four for the potentiostat) were fed through a flange through the vacuum chamber. All devices are computer-controlled and the automated process was triggered by the control system of ANTARES to synchronize the time equivalence of the NR, the filling process and the EIS measurements.

Filling process.-The electrolyte used is a $1 \mathrm{M}$ solution of $\mathrm{LiPF}_{6}$ in a mixture of ethylene carbonate (EC) and ethyl methyl carbonate (EMC; weight ratio EC:EMC of 3:7) with $2 \mathrm{wt} \%$ vinylene carbonate (VC). Generally for large-format cells multiple dosing iterations after absorption of the liquid by the cell assembly are required because of the little free space in the cell and the high flow resistance of the porous media. In this design, due to the little electrolyte amount (cell A: $3.4 \mathrm{~mL}$; cell B: $5.4 \mathrm{~mL}$ ) in comparison to the free volume between the flexible pouch foil and the stack, one dosing step was sufficient. As a result the filling process schematically mapped in Figure 3 was composed of five steps: evacuation, dosing, sealing, venting and wetting. The absolute pressure in the vacuum chamber was set to $50 \mathrm{mbar}$, the closing pressure of the sealing bars to $3 \mathrm{bar}$ for 3 seconds and the sealing temperature to $195^{\circ} \mathrm{C}$. After venting, the cell was wetted under ambient pressure for over 90 minutes.
Neutron radiography and image processing.-Before the experiments five dark field images were recorded with closed shutter obtaining the static noise of the detector. Then to secure a sufficient intensity of radiation on the detector behind the transmitted object, for each radiography, the neutron beam shutter was opened for 3 seconds at a neutron flux density of $6.4 \cdot 10^{7} \mathrm{~cm}^{-2} \mathrm{~s}^{-1}$. The electrolyte intake process into the pores of the cell stack was slow enough to not cause any notable motion blur in the image. The image acquisition procedure (exposure, sensor read-out and idle time) was repeated four times per minute recording a radiography of $1300 \times 2048$ pixels for the first 15 minutes. Afterwards the number of pictures taken was reduced to one image per minute. The effective size of one pixel was $73 \mu \mathrm{m}$ $\times 73 \mu \mathrm{m}$. Combined with the geometrical resolution, defined by the $\mathrm{L} \mathrm{D}^{-1}$ ratio of $500 \mu \mathrm{m}$, and the blurring due to the scintillator screen thickness of $100 \mu \mathrm{m}$ the spatial resolution resulted in approximately $150 \mu \mathrm{m}$.

The processing of all raw data was programmed in Matlab. The transmission images $T_{i}$ were calculated including the median of the dark field images $I_{\text {median, } d f}$, the intensity images of the cell $I_{i}$ and the reference intensity image $I_{r e f}$, which was recorded prior to filling:

$$
T_{i}=\frac{I_{i}-I_{\text {median }, d f}}{I_{\text {ref }}-I_{\text {median }, d f}}
$$

Each image was assigned the time stamp of the data file and the last recording without electrolyte visible was defined as the filling start. The images were cropped to the size of the cell stack and for this section of each image a grayscale threshold value with Otsu's method $^{27}$ was generated. Pixels $p x$ with a gray value smaller than the maximal threshold thresh $_{\text {max }}$ of all images, which resulted in 0.949, were counted representing the wetted area. The offset resulting from the number of pixels over this threshold value of the image prior to filling $(i=0)$ was substracted. The wetting degree $\phi_{i}$ was calculated by setting this difference proportional to the total amount of $2 \mathrm{D}$ pixels $(j=n)$ per detector image $i$ of NR:

$\phi_{i}$

$$
=\frac{\sum_{j=1}^{n}\left(\left\{p x_{i, j} \mid p x_{i, j}<\text { thresh }_{\text {max }}\right\}-\left\{p x_{0, j} \mid p x_{0, j}<\text { thresh }_{\text {max }}\right\}\right)}{\sum_{j=1}^{n} p x_{i, j}}
$$

Electrochemical impedance spectroscopy and analysis.-The electrochemical measurements were performed with an Interface $5000 \mathrm{E}$ potentiostat from Gamry Instruments. The sequence composed of open current potential (OCP) and EIS measurements was programmed to repeat itself over 90 minutes. First the OCP was measured over 15 seconds with a sample period of 0.5 seconds. Subsequently the potentiostatic EIS was started at an initial frequency of $100 \mathrm{kHz}$ to 1 $\mathrm{Hz}$ with 10 points per decade and an amplitude of $10 \mathrm{rms} \mathrm{mV}$ as AC excitation signal applied to the cell. The constant potential offset that 


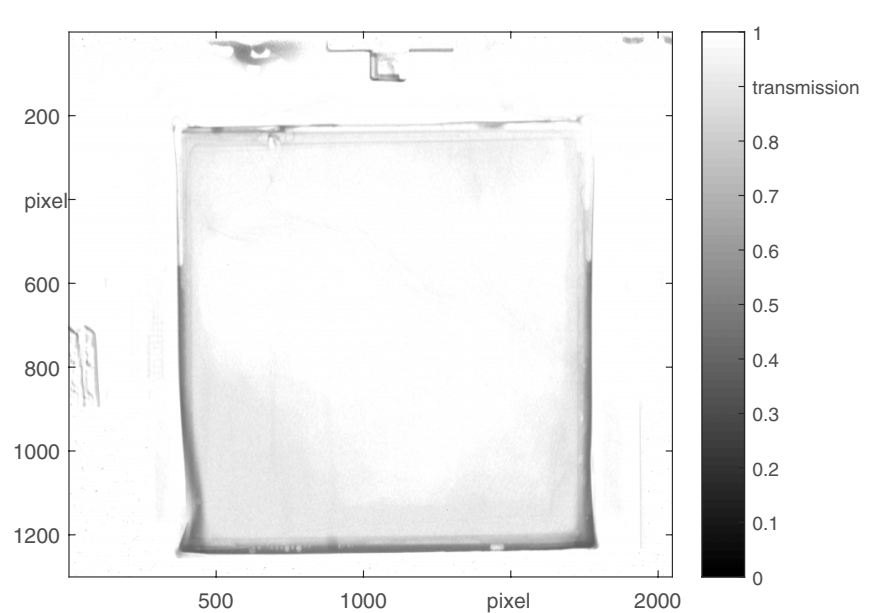

(a)

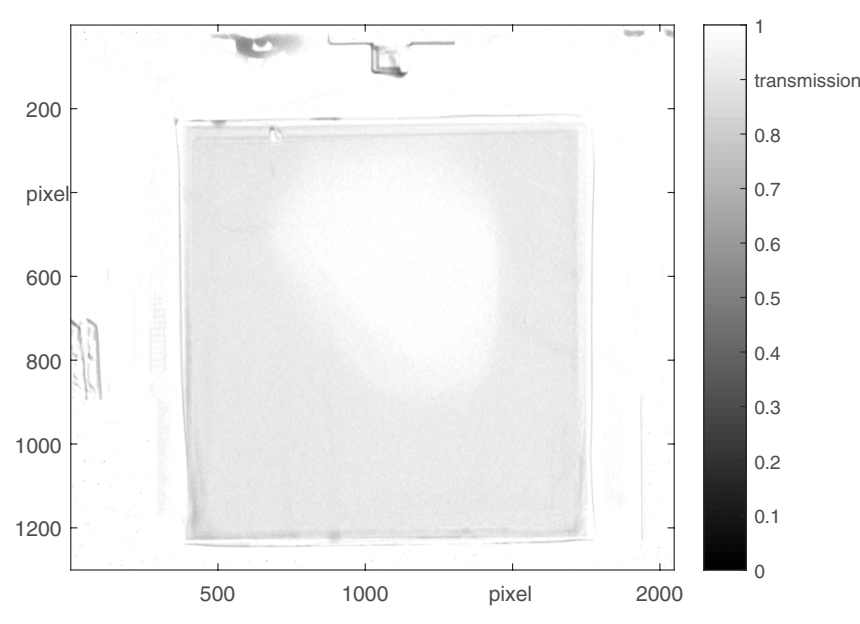

(c)

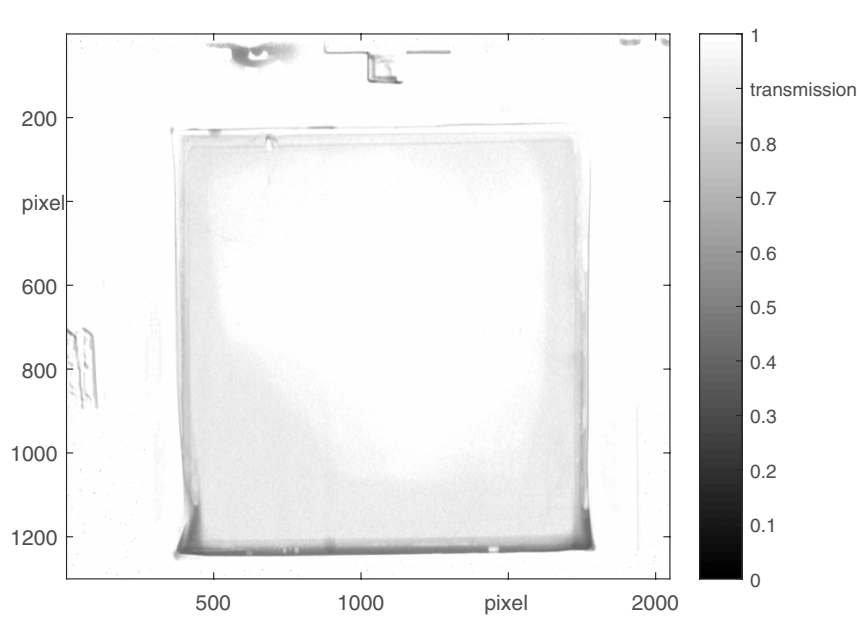

(b)

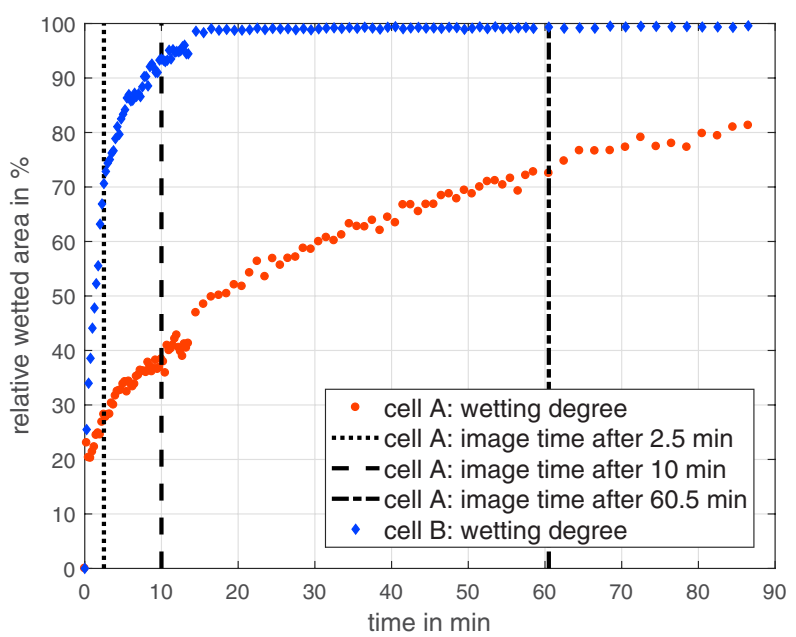

(d)

Figure 4. (a) - (c) NR images 1-3 of cell $\mathrm{A}$ at $2.5 \mathrm{~min}, 10 \mathrm{~min}$ and $60.5 \mathrm{~min}$ after dosing electrolyte, (d) Wetting degree of cell $\mathrm{A}$ (unstructured, $30 \%$ porosity) and cell $\mathrm{B}$ (structured, $30 \%$ porosity) over time.

can be applied to the cell throughout the data acquisition was set to zero versus the OCP of the cell. The AC voltage was summed with the DC voltage.

As with the image data from the NR, the processing of the electrochemical measurements was done in Matlab starting with the assignment of file timestamps. The EIS curves were analyzed searching for the data points nearest to the real axis and interpolating the zerocrossing which is shown in Figure 5c. By subtracting these points, the difference was calculated resulting in the shift of impedance along the real axis over time. In addition, the absolute value of the impedance was used to interpolate the frequency to the corresponding data pair with zero as the imaginary part.

\section{Results and Discussion}

Due to the small pore sizes, in the $\mu \mathrm{m}$ range, of electrodes and separators in comparison to the imaging resolution, the microscopic wetting of single pores could, as in Ref. 6 not be visualized. Nonetheless, the macroscopic wetting is a prerequisite for the uptake of liquid into the small pores. ${ }^{28}$ The wetting degree $\phi_{i}$ is defined as the relative wetted area over time. Figure 4 shows the NR images of cell A at three times with the corresponding wetting degree as well as the wetting degree of cell $\mathrm{B}$. The gray area of the cross section of the cell stack increases from Figures $4 \mathrm{a}$ to $4 \mathrm{c}$ at the expense of the white space, while the dark section at the edges of the stack shrinks.
As mentioned above, deeper gray values represent a lesser intensity of neutrons transmitted through the pouch bag cell and thus, a larger amount of electrolyte in the corresponding cell section. These dark sections at the cell stack edges can be interpreted as electrolyte bulk as, due to sealing under vacuum and subsequent venting of the chamber, the liquid gets pressed into the remaining space of the pouch bag pocket. From there, the cell assembly soaks up the electrolyte causing a more homogeneous distribution of the electrolyte liquid over time. This effect is visualized by the wetting degree in Figure 4d. The deceleration of the wetting rate over time is observable. After 90 minutes, over $80 \%$ at the cross section of the cell stack is wetted. Cell B shows a different wetting behavior. Here the mean cross-section area is completely wetted after 15 minutes.

In Figure 5 (a left), Nyquist plots of the EIS responses of cell A at the three wetting instants of the shown NR pictures are displayed. The metering time period for one EIS measurement is dependant on the covered frequency range, causing a small distortion within each curve. However, in comparison to the total wetting time the duration of one EIS measurement sweep, which amounted to 3.86 seconds, can be neglected.

Comparing one EIS curve during the filling process shown in Figure $5 \mathrm{a}$ with the schematic curve from Figure 2 in the Nyquist plot the assumption made in the beginning can be discussed. The first semi-circle flattens into a plateau and from there the transition into a strong rise is visible. Either it is not possible to distinguish the second 


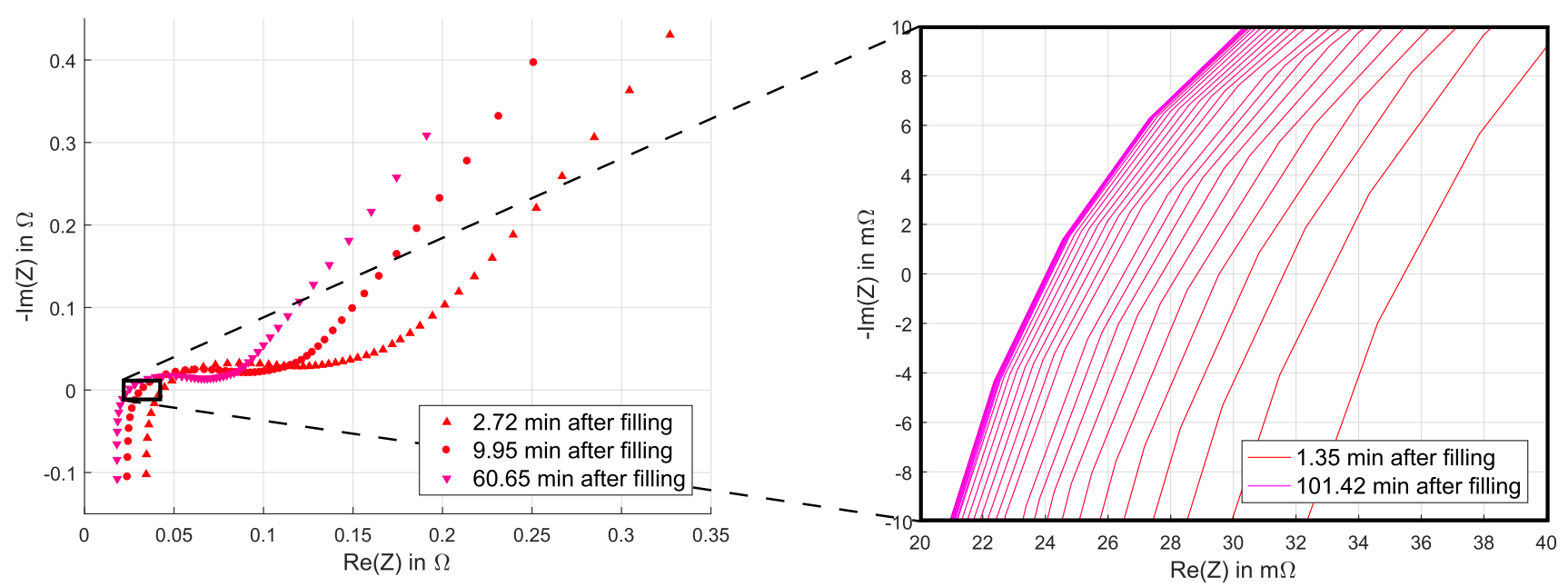

(a)

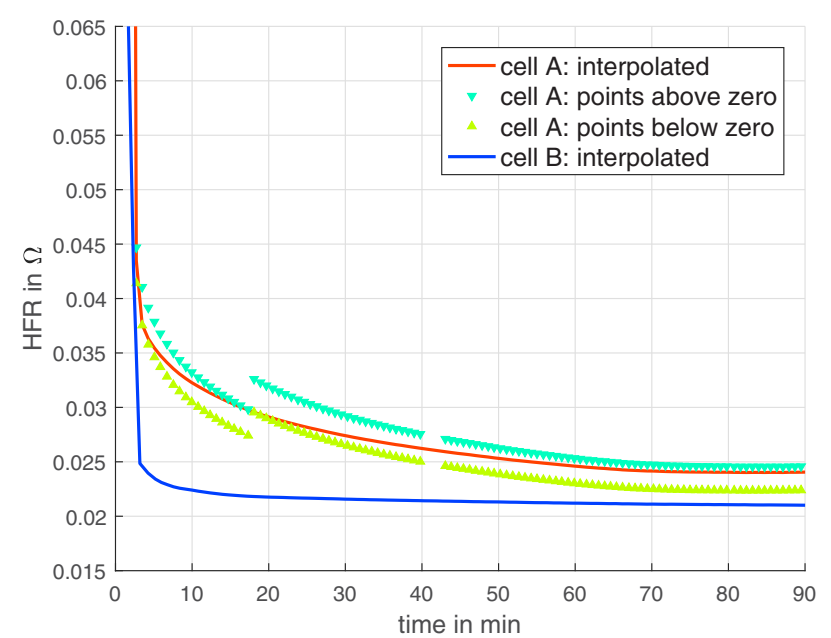

(b)

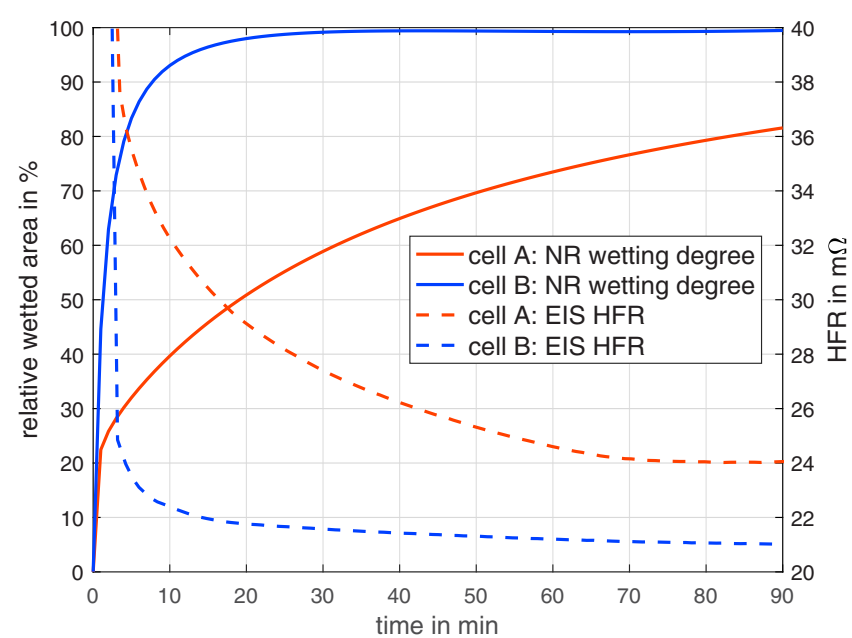

(c)

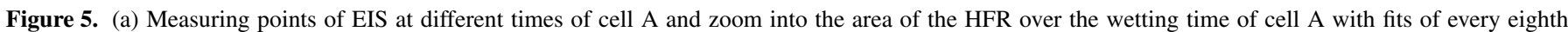

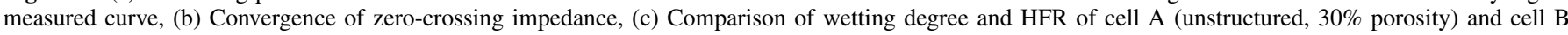
(structured, $30 \%$ porosity).

semi-circle even though the SOC is $0 \%$, or the rise is not the section for the solid-state diffusion at frequencies higher as $1 \mathrm{~Hz}$ but the beginning of the second semi-circle during the filling process. Eftekhari presents a similar impedance model without second semi-circle due to the missing SEI in supercapacitors. ${ }^{22}$ When evaluating the separate EIS curves over time, a shift to smaller impedance values along the real axis can be observed. On closer inspection of the intersection, which is the point without phase shifting and thereby represents the ohmic part of the internal cell resistance (Figure 5 (a right)), the distance between the interpolated impedance curves is decreasing with wetting time. The absolute values of this HFR over time are displayed in Figure $5 \mathrm{~b}$ for cell $\mathrm{A}$ and cell $\mathrm{B}$. The connection between wetting degree, calculated from the NR images, and the EIS signal becomes apparent in Figure 5c. As the wetting degree increases, the HFR decreases. It is possible to assign each wetting degree over time to a corresponding HFR value for each cell. For example, an HFR value of $34 \mathrm{~m} \Omega$ corresponds to a wetting degree of $37 \%$ for cell A, while the same HFR is correlated to $70 \%$ wetting for cell B. Possible reasons herefore are the differences in the designs of the electrodes. The laser-structured electrodes of cell B not only influence the wettability of the cell, but also its electrochemical behavior by ablating $10 \%$ of the active material compared to cell A. Also, deviations between visual wetting and true wetting are caused by the measurement methods. With neutron radiography, it is possible to detect the macroscopic wetting instanta- neously wherever electrolyte liquid is present. In contrast, EIS detects the microscopic wetting as the electrolyte closes the circuit: the liquid must not only penetrate between the layers of the components, but also into the pores. This infiltration requires more time after dosing, delaying the detection of significant measuring points. For the necessary time before charging the cells in production, this microscopic wetting is of high importance. Nevertheless, Figure 5c shows that the macroscopic wetting, i.e. transporting electrolyte to the pores, is a requirement for and connected to the microscopic wetting. Only with converging wetting degree the HFR starts to slow its decrease. With faster wetting (cell B), the HFR converges faster as well in comparison to a slow wetting (cell A).

The change of the HFR over time visualized in Figure 6a is defined as

$$
H \dot{F} R(t)=\frac{d H F R}{d t}
$$

and can be correlated to the wetting velocity. As the wetting and the HFR converges, the $H \dot{F} R$ approaches $0 m \Omega \min ^{-1}$. For cell $\mathrm{B}$, the $H \dot{F} R$ after 30 minutes is low enough to ensure sufficient wetting, while for cell A, the change of the HFR is only after 80 minutes on the same level. Furthermore, the correlating wetting degree of approximately $80 \%$ indicates an insufficient dosing volume of electrolyte liquid (see Filling process section). By comparing the last taken NR 


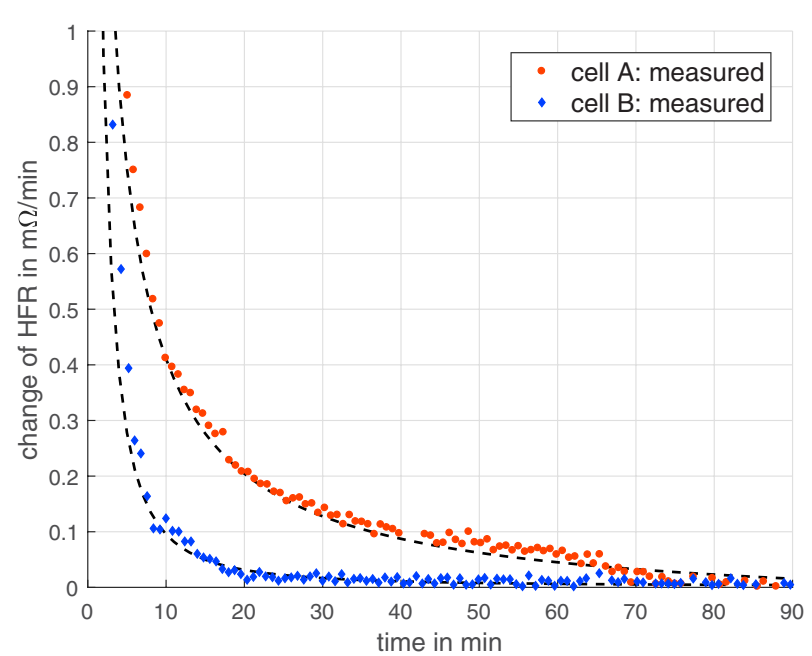

(a)

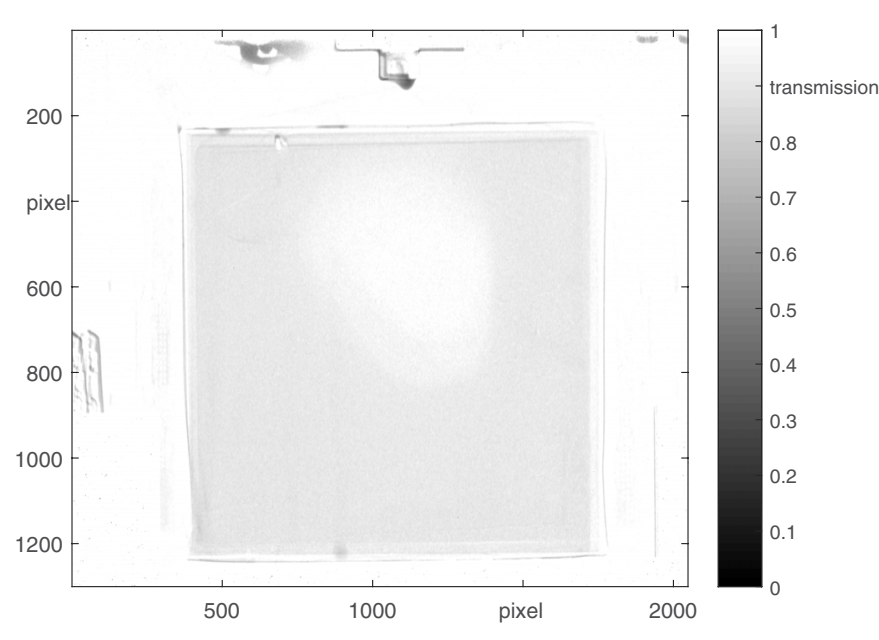

(b)

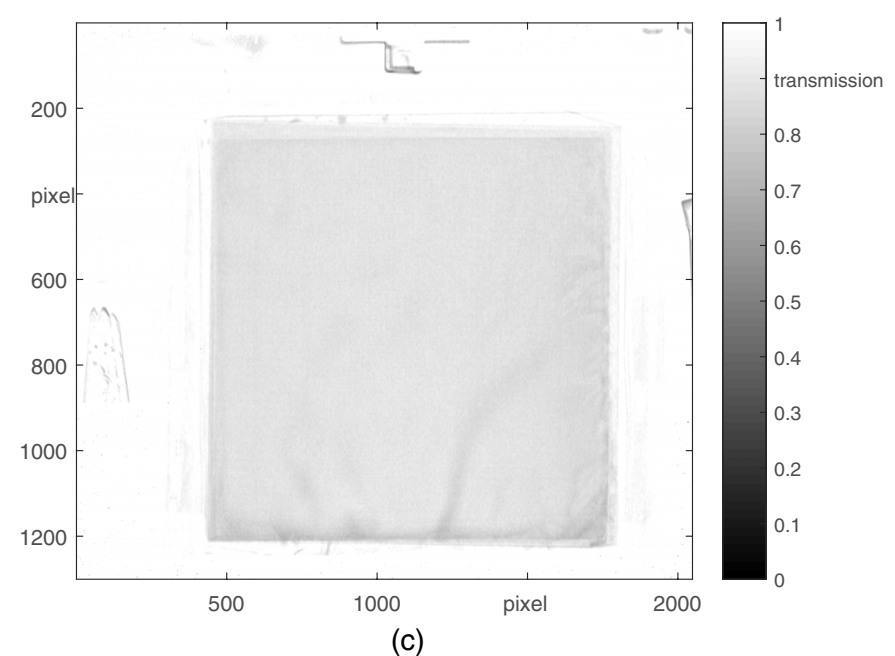

Figure 6. (a) Convergence of $H \dot{F} R$ of cell A (unstructured, 30\% porosity) and cell B (structured, 30\% porosity) (b) NR image of cell A at 86.5 min after dosing electrolyte (c) NR image of cell B at 86.5 min after dosing electrolyte.

images of both cells in Figures $6 \mathrm{~b}-6 \mathrm{c}$, it can be seen that cell B is completely wetted and has even darker gray values than cell $\mathrm{A}$. The image of cell A neither shows remaining electrolyte bulk in the cell nor gray value gradients in the wetted area.

Thus, the change of the HFR can be used as a criterion for the wetting state and as a consequence, for the necessary wetting time in production. We used a format consisting of only 5 anodes and 4 cathodes securing the NR image quality for these first measurements. With larger formats, the number of cell layers as well as the surface area per layer are likely to increase, which will affect the intrusion rate of the electrolyte into the pores of the active materials. This growing wetting duration is estimated to stretch all curves in Figure $6 \mathrm{c}$ along the time axis.

In Figure 7a the frequencies of the excitation signals, which correspond to the interpolated HFR are displayed. The interpolation converges for cell $\mathrm{A}(\mathrm{B})$ to the frequency of $13.66(10.35) \mathrm{kHz}$ and shows no changes in inductive reactances of the cell components. Together with the OCP voltage, it is an indicator for stable measurements as well as stable electrochemical behavior of the cells with progressing wetting. As earlier mentioned, it is important not to trigger electrochemical reactions leading to the formation of the cell before completion of wetting. Those reactions let the SOC increase and therefore, the OCP as well. ${ }^{12}$ Furthermore, SOC, temperature and current amplitude affect the battery resistance. ${ }^{13}$ With longer pulse lengths and higher signal amplitudes the risk of unintentionally charging the cell and thereby starting the formation process increases. Compared to DC pulses, the signal amplitude used in EIS is very low. ${ }^{14}$ Therefore, methods based on DC pulses are detrimental as a measurement method in production after dosing the electrolyte and before cycling the cells for the first time. Here the OCP is a continuous function over time, which is a promising indicator for the method not to influence the cell chemistry through unintended charging or electrochemical side reactions, which could have a negative impact on product quality of the cells after formation. Although the OCP is decreasing over time, this change had no influence on the AC response signals of the EIS measurements, because the constant potential offset of the EIS compared to the OCP was set to zero.

Using EIS as a measurement method for the wetting degree, the additional effort in the industrial production of LIB is limited: For formation, cells have to be electrically contacted to the charging/discharging station. Either every cell could be contacted right after filling for EIS measurements and automatically charged after wetting is completed or only random samples are checked to ensure the right wetting duration of the batch. Both approaches promise a great advantage in cost and quality for the industry, if the investment in EIS hardware pays off in terms of production volume. At least, when designing processes for new cell formats, EIS is a helpful method for the analysis of wetting within the cell. 


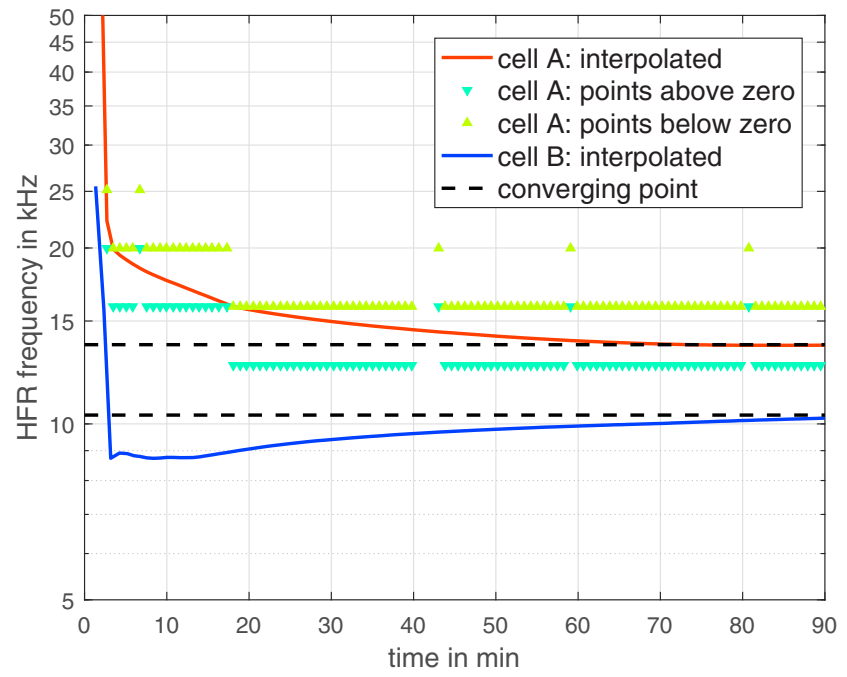

(a)

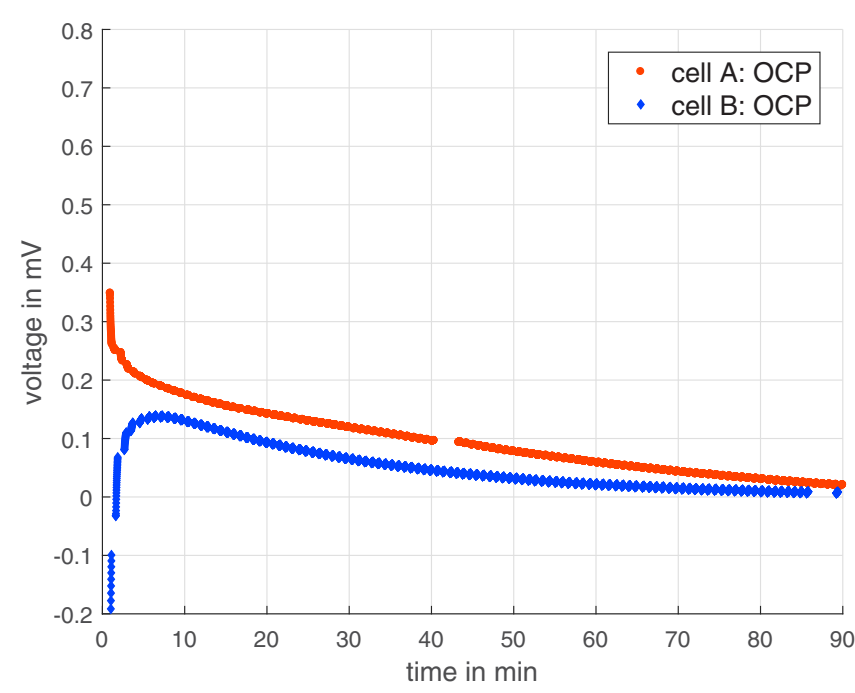

(b)

Figure 7. (a) Convergence of zero-crossing frequency, (b) Convergence of OCP of cell A (unstructured, 30\% porosity) and cell B (structured, $30 \%$ porosity).

\section{Conclusions}

It has been shown that the electrochemical impedance changes during the filling process of LIB in the production. In detail, the HFR is directly linked to the wetting state of the cell. This has been determined by comparing the response signal of EIS to radiography images during the filling process on cells with completely different wetting behavior. Thus the output signal of EIS can be used to measure the wetting progress during the filling process. Furthermore, the HFR signal varies over the wetting time and converges as the EIS signal of the LIB shifts to smaller impedances along the real axis. It was possible to produce stable measurements without influencing the cells by charging or other means. As a result, the concept of using the observed correlation to directly determine the minimum time required per cell for wetting in production is proposed. It should be mentioned that the experiments are not enough evidence to draw quantifiable conclusions. Therefore, future research of the authors will focus on quantification of this effect and the reliability of the method to ensure a high accuracy and stability of the measurements. Especially important is to ensure that the EIS has no impact on the electrochemical performance of the cell after cycling triggered by the AC signal over the wetting duration. The scale up to industrial relevant large cell sizes is another necessary step to enable the method for the battery production. It is probable that the method is even more suitable for large formats because of greater measurable differences in the impedance and a longer time duration of the wetting.

All in all electrochemical impedance is a possible method to measure the wetting degree of lithium-ion batteries in the cell production. First measurements coupled with images by neutron radiography show promising results for upcoming experiments and a possible industrial application.

\section{Acknowledgments}

The experiments were conducted at MLZ at the Technical University of Munich. The support and beam time of MLZ is gratefully acknowledged. Moreover, the authors are gratefully indebted to the German Federal Ministry of Education and Research (BMBF) for funding their research within the project ExZellTUMII (grant number 03XP0081). The authors thank Johannes Landesfeind and Tanja Zünd for their critical feedback on the EIS interpretation and Gamry Instruments for the loan of the potentiostat.

\section{ORCID}

Florian J. Günter (10) https://orcid.org/0000-0002-5967-6801

Jan Bernd Habedank (D) https://orcid.org/0000-0003-2057-7037

Tobias Neuwirth (10) https://orcid.org/0000-0002-9858-1194

\section{References}

1. B. Scrosati and J. Garche, Lithium batteries: Status, prospects and future, Journal of Power Sources, 195(9), 2419 (2010).

2. D. Andre, S.-J. Kim, P. Lamp, S. F. Lux, F. Maglia, O. Paschos, and B. Stiaszny, Future generations of cathode materials: An automotive industry perspective, Journal of Materials Chemistry A, 3(13), 6709 (2015).

3. T. Knoche and G. Reinhart, Electrolyte filling of large-scale lithium-ion batteries: Main influences and challenges for production technology, Applied Mechanics and Materials, 794, 11 (2015).

4. D. L. Wood, J. Li, and C. Daniel, Prospects for reducing the processing cost of lithium ion batteries, Journal of Power Sources, 275, 234 (2015).

5. W. J. Weydanz, H. Reisenweber, A. Gottschalk, M. Schulz, T. Knoche, G. Reinhart, M. Masuch, J. Franke, and R. Gilles, Visualization of electrolyte filling process and influence of vacuum during filling for hard case prismatic lithium ion cells by neutron imaging to optimize the production process, Journal of Power Sources, $\mathbf{3 8 0}$, 126 (2018)

6. T. Knoche, V. Zinth, M. Schulz, J. Schnell, R. Gilles, and G. Reinhart, In situ visualization of the electrolyte solvent filling process by neutron radiography, Journal of Power Sources, 331, 267 (2016).

7. K. W. Herwig, Introduction to the neutron, in: H. Z. Bilheux, R. McGreevy, and I. S. Anderson (Eds.); Neutron Imaging and Applications, Neutron Scattering Applications and Techniques, Springer-Verlag, s.1., 2009, pp. 3.

8. V. F. Sears, Neutron scattering lengths and cross sections, Neutron News, 3(3), 26 (2006).

9. M.-S. Wu, T.-L. Liao, Y.-Y. Wang, and C.-C. Wan, Assessment of the wettability of porous electrodes for lithium-ion batteries, Journal of Applied Electrochemistry, 34(8), 797 (2004).

10. V. F. Lvovich, Fundamentals of electrochemical impedance spectroscopy, in: V. F. Lvovich (Ed.), Impedance spectroscopy, Vol. 3, Wiley, Hoboken, N.J, pp. 1, 2012.

11. B. V. Ratnakumar, M. C. Smart, and S. Surampudi, Electrochemical impedance spectroscopy and its applications to lithium ion cells, in: 2002 the Seventeenth Annual Battery Conference on Applications and Advances, I E E E, Piscataway, Jan. 2002, pp. 273.

12. D. Andre, M. Meiler, K. Steiner, C. Wimmer, T. Soczka-Guth, and D. U. Sauer, Characterization of high-power lithium-ion batteries by electrochemical impedance spectroscopy. i. experimental investigation, Journal of Power Sources, 196(12), 5334 (2011).

13. N. Omar, M. Daowd, P. van den Bossche, O. Hegazy, J. Smekens, T. Coosemans, and J. van Mierlo, Rechargeable energy storage systems for plug-in hybrid electric vehicles-assessment of electrical characteristics, Energies, 5(8), 2952 (2012).

14. A. Barai, K. Uddin, W. D. Widanage, A. McGordon, and P. Jennings, A study of the influence of measurement timescale on internal resistance characterisation methodologies for lithium-ion cells, Scientific reports, 8(1), 21 (2018). 
15. J. Landesfeind, D. Pritzl, and H. A. Gasteiger, An analysis protocol for three-electrode li-ion battery impedance spectra: Part i. analysis of a high-voltage positive electrode, Journal of The Electrochemical Society, 164(7), A1773 (2017).

16. M. D. Levi, K. Gamolsky, D. Aurbach, U. Heider, and R. Oesten, On electrochemical impedance measurements of lixco0.2ni0.8o2 and lixnio2 intercalation electrodes, Electrochimica Acta, 45(11), 1781 (2000). URL http://www.sciencedirect.com/science/article/pii/S0013468699004028.

17. J. Landesfeind, J. Hattendorff, A. Ehrl, W. A. Wall, and H. A. Gasteiger, Journal of The Electrochemical Society, 163(7), A1373 (2016).

18. G. Ning, B. Haran, and B. N. Popov, Capacity fade study of lithium-ion batteries cycled at high discharge rates, Journal of Power Sources, 117(1-2), 160 (2003).

19. J. Li, E. Murphy, J. Winnick, and P. Kohl, Studies on the cycle life of commercial lithium ion batteries during rapid charge-discharge cycling, Journal of Power Sources, 102(1-2), 294 (2001).

20. P. Vyroubal and T. Kazda, Equivalent circuit model parameters extraction for lithium ion batteries using electrochemical impedance spectroscopy, Journal of Energy Storage, 15, 23 (2018).

21. J. Song and M. Z. Bazant, Effects of nanoparticle geometry and size distribution on diffusion impedance of battery electrodes, Journal of the Electrochemical Society, 160(1), A15 (2012).
22. A. Eftekhari, The mechanism of ultrafast supercapacitors, Journal of Materials Chemistry A, 6(7), 2866 (2018).

23. T. Günther, N. Billot, J. Schuster, J. Schnell, F. B. Spingler, and H. A. Gasteiger, The manufacturing of electrodes: Key process for the future success of lithium-ion batteries, Advanced Materials Research, 1140, 304 (2016).

24. T. Knoche, F. Surek, and G. Reinhart, A process model for the electrolyte filling of lithium-ion batteries, Procedia CIRP, 41, 405 (2016).

25. J. B. Habedank, F. J. Guenter, N. Billot, R. Gilles, T. Neuwirth, G. Reinhart, and M. F. Zäh, Rapid electrolyte wetting in li-ion pouch cells with laser structured electrodes observed by neutron radiography, submitted.

26. E. Calzada, F. Gruenauer, M. Mühlbauer, B. Schillinger, and M. Schulz, New design for the antares-ii facility for neutron imaging at frm ii, $\mathrm{Nu}$ clear Instruments and Methods in Physics Research Section A: Accelerators, Spectrometers, Detectors and Associated Equipment, 605(1-2), 50 (2009).

27. N. Otsu, A threshold selection method from gray-level histograms, IEEE Transactions on Systems, Man, and Cybernetics, 9(1), 62 (1979).

28. Y. Sheng, Investigation of electrolyte wetting in lithium ion batteries: Effects of electrode pore structures and solution, Dissertation, University of Wisconsin-Milwaukee, Wisconsin (2015). 